\title{
烧结温度对硼硅基质荧光玻璃发光性能影响
}

\author{
李 杨 ${ }^{1,2,3}$, 胡丽丽 ${ }^{1}$, 杨波波 ${ }^{4}$, 石明明 ${ }^{4}$, 邹 军 ${ }^{4}$
}

(1. 中国科学院 上海光学精密机械研究所 高功率激光单元技术研发中心, 上海 201800; 2. 中国科学院大学, 北 京 $100049 ; 3$. 上海应用技术大学 材料科学与工程学院, 上海 $201418 ; 4$. 上海应用技术大学 理学院, 上海 201418)

摘 要: 采用共烧结法制备了嗍硅基质 $\mathrm{Ce}: \mathrm{YAG}$ 荧光玻璃,研究了烧结温度在 $600^{\circ} \mathrm{C} \sim 900^{\circ} \mathrm{C}$ 范围内, $\mathrm{Ce}$ : $\mathrm{YAG}$ 苂光玻 璃的发光强度变化和色坐标漂移规律。结果表明, 随着烧结温度的升高, Ce: YAG 苂光玻璃发光强度先增强后减弱, $700^{\circ} \mathrm{C}$ 烧结时, 荧光玻璃获得最大发光强度; 超过 $850^{\circ} \mathrm{C}$ 烧结时, 荧光玻璃无发光性能; 同时, 色坐标 $(x, y)$ 发生漂移, 且比相同烧结温度的荧光粉漂移幅度大。通过 X 射线粉末衍射仪、差示扫描量热分析仪和 X 射线光电子能谱分析 仪测试分析表明: 随着烧结温度升高, 荧光粉中的 $\mathrm{Ce}^{3+}$ 被玻璃基质氧化成 $\mathrm{Ce}^{4+}$, 玻璃液体腐蚀破坏了菼光粉 $\mathrm{YAG}$ 晶体结构, 降低了苂光玻璃的发光强度, 从而导致色坐标劣化漂移。

关＼cjkstart键＼cjkstart词: Ce: YAG 苂光玻璃; 发光强度; 色坐标漂移; 烧结温度

中图分类号: TB383 文献标识码: A

\section{Effect of Sintering Temperature on Luminescence Properties of Color Conversion Glasses in Borosilicate Glasses}

\author{
LI Yang ${ }^{1,2,3}$, HU Li-Li ${ }^{1}$, YANG Bo-Bo ${ }^{4}$, SHI Ming-Ming ${ }^{4}$, ZOU Jun $^{4}$
}

(1. Key Laboratory of Materials for High Power Laser, Shanghai Institute of Optics and Fine Mechanics, Chinese Academy of Sciences, Shanghai 201800, China; 2. University of Chinese Academy of Sciences, Beijing 100049, China; 3. School of Material Science and Engineering, Shanghai Institute of Technology, Shanghai 201418, China; 4. School of Science, Shanghai Institute of Technology, Shanghai 201418, China)

\begin{abstract}
The color conversion glass is a promising material for light emitting diodes encapsulation due to its high thermal conductivity and good thermal stability. In present study, color conversion glasses were prepared by sintering a mixture of borosilicate glass frits and a YAG phosphor at temperature from $600^{\circ} \mathrm{C}$ to $900^{\circ} \mathrm{C}$. The effects of sintering temperature on luminescence properties were examined. Characteristics of color conversion glasses, with respect to luminescence intensity, CIE (Commission International de I'Eclairage) chromaticity and CCT (Correlated Color Temperature) were analyzed based on luminescence excitation and emission spectra. These characteristics are dependent on sintering temperature of the color conversion glasses. The CIE coordinates shift with increase of sintering temperature. The glass, sintered at $700^{\circ} \mathrm{C}$, shows the best luminescence properties. But for the glasses sintered above $850^{\circ} \mathrm{C}$, their luminescence properties may be destroyed. Test results of X-ray diffraction (XRD), differential scanning calorimetry (DSC) and X-ray photoelectron spectroscopy (XPS) reveal that degradation of luminescence properties with increase of sintering temperature is ascribed to the lattice disturbance of YAG and oxidation of $\mathrm{Ce}^{3+}$, which is caused
\end{abstract}

收稿日期: 2016-06-15; 收到修改稿日期：2016-09-06

基金项目：国家自然基金青年基金(51302171); 上海市科委学科能力建设项目(14500503300); 上海联盟计划项目(LM201547) National Natural Science Foundation of China (51302171); Science and Technology Commission of Shanghai Municipality (14500503300); Shanghai Municipal Alliance Program (LM201547)

作者简介: 李 杨(1982-), 女, 博士研究生, 实验师. E-mail: liyang123@sit.edu.cn

通讯作者: 胡丽丽, 研究员. E-mail: hulili@siom.ac.cn 
by reactions between glass matrix and YAG phosphors. The present study may promote the application of color conversion glasses in light emitting diodes encapsulation.

Key words: Ce: YAG color conversion glass; luminescence intensity; CIE coordinates shift, sintering temperature

白光发光二极管(LED)因其节能、环保、寿命 长等优点而被广泛使用在照明和车灯等领域 ${ }^{[1-3]}$ 。目 前，商业用的大功率白光 LED 光源封装，主要采用 硅胶混合荧光粉粘接在蓝光芯片上 ${ }^{[4]}$ 。但是，硅胶为 有机物, 其玻璃转变温度 $\left(T_{\mathrm{g}}\right)$ 只有 $150^{\circ} \mathrm{C}$ 左右, 且热导 率低, 在光源使用过程中极易出现老化而变黄 ${ }^{[5]}$ 。硅 胶老化会造成封装结构不稳定, 苂光粉和芯片粘接 不牢, 也易出现压断焊接金线而造成死灯现象, 硅 胶变黄会造成光源色温漂移, 以上不稳定因素极大 影响了 LED 光源可靠性和使用寿命, 限制了大功率 LED 的发展。苂光玻璃和苂光玻璃陶瓷, $T_{\mathrm{g}}$ 较高, 具 有高稳定性和高热导率, 可以有效解决硅胶封装 LED 的可靠性问题 ${ }^{[6]}$ 。低熔点玻璃与菼光粉共烧结 制备苂光玻璃的方法, 因其烧结温度低, 可有效保护 苂光粉性能, 制备成本低而被广泛采用 ${ }^{[7]}$ 。目前, 常 用的低温玻璃基质有铅硅玻璃 ${ }^{[8]}$ 、碲酸盐玻璃 ${ }^{[9]}$ 、 磷酸盐玻璃 ${ }^{[10]}$ 、硅酸盐玻璃 ${ }^{[11]}$ 、碀硅酸盐玻璃 ${ }^{[12]}$ 等。根据欧盟 CE-ROHS 标准 ${ }^{[13]}$, 制备光电器件时 铅含量需严格控制低于 $1000 \times 10^{-6}$, 因此铅硅玻璃 基质应用前景受限; 碲酸盐玻璃的 $T_{\mathrm{g}}$ 低, 光学性能 好，但是机械强度差极大限制了其实际应用前景； 磷酸盐玻璃易水解, 会降低苂光粉的发光性能; 硅 酸盐玻璃烧结温度高, 致使烧结工艺难度增大; 而 磀硅酸盐玻璃良好的机械性能保证了荧光玻璃在 LED 封装过程中的可操作性。

本工作选用硼硅酸盐玻璃基质与 $\mathrm{Ce}: \mathrm{YAG}$ 苂光 粉共烧结制备苂光玻璃, 烧结温度选择 $600^{\circ} \mathrm{C} \sim 900^{\circ} \mathrm{C}$, 保温时间 $20 \mathrm{~min}$ 。研究不同的烧结温度对发光强度 和色坐标的影响, 并研究了在较高烧结温度下, 苂 光玻璃发光性能降低的原因。

\section{1 实验方法}

\section{1 样品制备}

玻璃基质组分为 $\mathrm{B}_{2} \mathrm{O}_{3}-\mathrm{SiO}_{2}-\mathrm{ZnO}-\mathrm{BaO}-\mathrm{Na}_{2} \mathrm{O}$, 采 用传统的熔融冷却法制备, 熔制温度 $1100^{\circ} \mathrm{C}$ 。将制 备好的基质玻璃磨成粉与 $3 \mathrm{wt} \%$ 荧光粉(威士玻尔, 型号 YAP4454-L)混合均匀, 放入马弗炉中共烧结, 烧结温度 $600 \sim 900^{\circ} \mathrm{C}$, 保温 $20 \mathrm{~min}$, 最后将制成的 苂光玻璃研磨抛光成 $1 \mathrm{~mm}$ 厚的样品。

\section{2 样品表征}

采用日本 Rigaku 的 Ultima IV 型 X 射线衍射仪 $(\mathrm{Cu} \mathrm{Ka}, \lambda=1.5406 \mathrm{~nm})$ 对荧光玻璃的晶相组成进行 分析, 衍射角度 $2 \theta=10^{\circ} \sim 60^{\circ}$, 扫描速率 $0.02^{\circ} /$ step 和 $4 \%$ min。采用 Edinburgh 苂光光谱仪测试样品的 激发光谱和荧光光谱, 氙灯泵浦。采用德国 $\mathrm{NETZSCH}$ 公司的 STA449 型差热分析仪对样品进 行热分析。采用赛默飞舍尔 Escalab 250Xi X 射线光 电子能谱分析仪测试 $\mathrm{Ce}^{3+}$ 和 $\mathrm{Ce}^{4+}$ 的含量。采用阿基 米德法测量荧光玻璃的密度。采用天勤公司 SVS2010-M-3D 影像测量仪检测玻璃内气泡。

\section{2 结果与分析}

\section{1 发光性能研究}

图 1 为不同温度下保温烧结 $20 \mathrm{~min}$ 得到的苂光 粉和苂光玻璃的激发谱和发射谱。从图 1(a,b)中可 以看出, 随着烧结温度的升高, 苂光粉的激发峰值 强度和发射峰值强度没有明显的变化, 说明在空气 中 $900^{\circ} \mathrm{C}$ 保温烧结 $20 \mathrm{~min}$, 对苂光粉的激发峰值强 度和发射峰值强度没有明显的影响。从图 1(c,d)中 可以看出，苂光玻璃的激发光谱和发射光谱与苂光 粉基本相似，只是在 $340 \mathrm{~nm}$ 的激发峰强度比苂光粉 低，源于玻璃基质在此波段的吸收。苂光玻璃激发 谱在 $340 \mathrm{~nm}$ 和 $460 \mathrm{~nm}$ 处的峰值强度先增加后减小, 烧结温度为 $670^{\circ} \mathrm{C}$ 时, 激发峰值强度最强; 烧结温 度超过 $850^{\circ} \mathrm{C}$ 时，激发谱没有谱峰。随着烧结温度的 升高, 发射谱在 $560 \mathrm{~nm}$ 处的峰值强度先增加后减 小, 烧结温度在 $700^{\circ} \mathrm{C}$ 时, 发射峰值强度最强, 超过 $850^{\circ} \mathrm{C}$ 时, 发射谱没有谱峰。当烧结温度低于 $670^{\circ} \mathrm{C}$ 时, 玻璃内可能存在大量的气泡降低了蓝光激发和 黄光发射。当烧结温度超过 $850^{\circ} \mathrm{C}$ 时, 玻璃基质完全 破坏了 $\mathrm{Ce}$ : YAG 苂光粉的发光性能。

为了能精确显示苂光玻璃的发光颜色变化，对 比分析了不同温度保温烧结 $20 \mathrm{~min}$ 得到的苂光玻 璃和荧光粉的色坐标变化, 如图 2 所示。由图 2(a) 可以看出, 随着烧结温度的升高, 荧光粉色坐标 CIE- $y$ 值先变小后变大, 当烧结温度为 $700^{\circ} \mathrm{C}$ 时, 达到最小; CIE- $x$ 值先增加后减小, 在烧结温度为 $700^{\circ} \mathrm{C}$ 时, 达到最大。由图 2(b)可看出, 当烧结温度 
高于 $700^{\circ} \mathrm{C}$ 时, 随着烧结温度的升高, 荧光玻璃的 色坐标变化规律与苂光粉基本一致, 但是当烧结温 度低于 $700^{\circ} \mathrm{C}$ 时, 苂光玻璃的色坐标变化规律与荧 光粉不同。为了直观显示荧光玻璃与荧光粉之间发 光颜色的变化差异，进一步对比分析了不同温度烧 结的苂光玻璃和荧光粉色温差值的变化规律, 如图 3 所示。随着烧结温度的升高, 荧光玻璃和荧光粉之
间的色温差值从 $-241 \mathrm{~K}$ 到 $+105 \mathrm{~K}$ 线性增加, 当烧结 温度为 $700^{\circ} \mathrm{C}$ 时, 荧光玻璃与荧光粉的色温值最接 近; 当烧结温度低于 $700^{\circ} \mathrm{C}$ 时，苂光玻璃的色温低 于苂光粉可能源于玻璃内大量气泡散射损失了一部 分蓝光, 如图 3 插图示出了 $600^{\circ} \mathrm{C}$ 烧结的玻璃内气 泡; 当烧结温度高于 $700^{\circ} \mathrm{C}$ 时，苂光玻璃中 $560 \mathrm{~nm}$ 处 的发射峰值降低，导致其色温比荧光粉高。
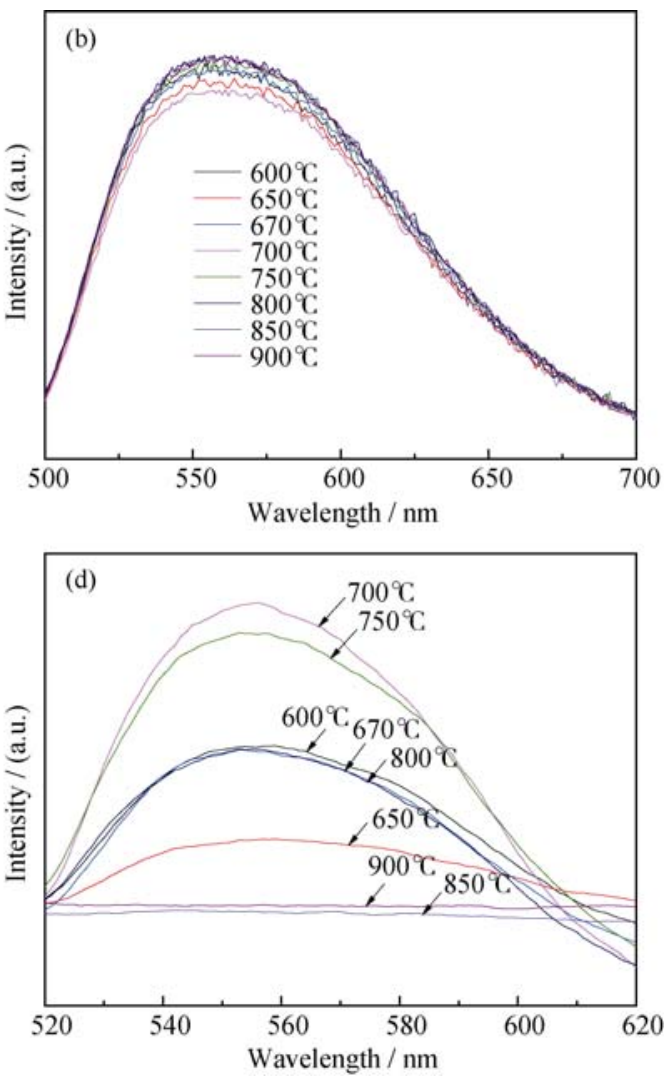

图 1 不同温度烧结的苂光粉和苂光玻璃的激发谱和发射谱

Fig. 1 PLE and PL spectra of their phosphors and their color conversion glasses sintered at different temperatures (a) PLE of the phosphors; (b) PL of the phosphors; (c) PLE of the color conversion glasses; (d) PL of the color conversion glasses
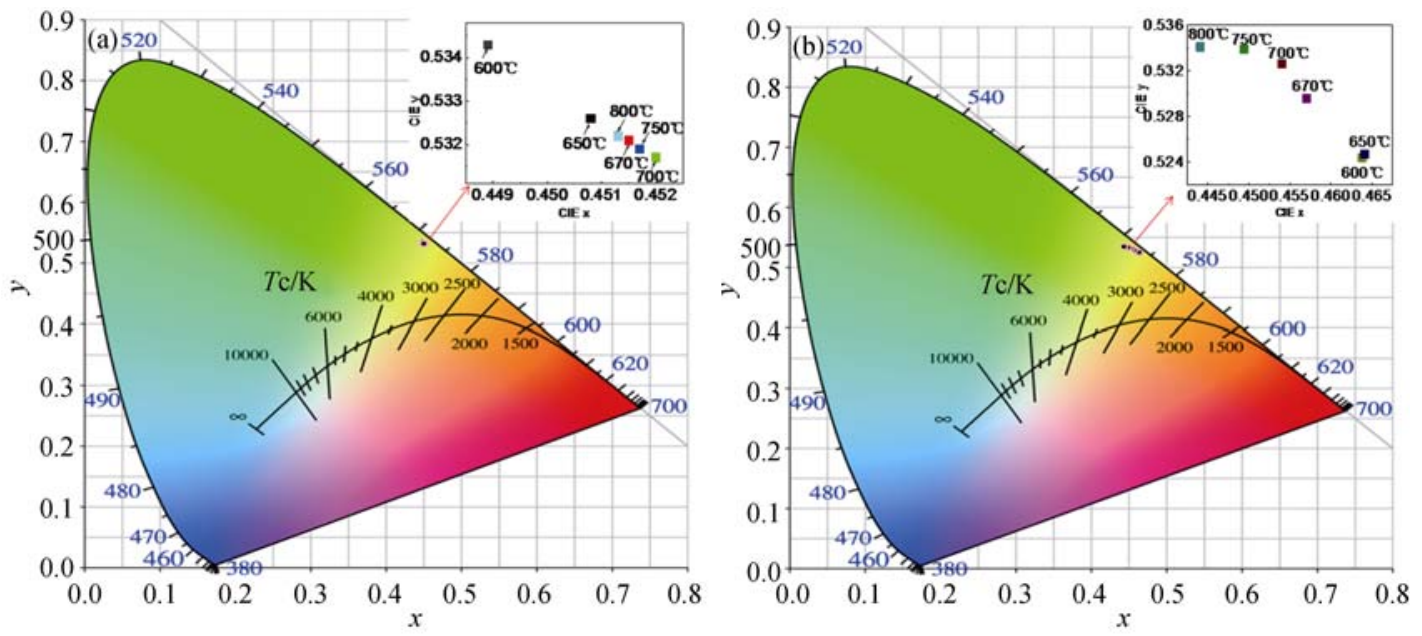

图 2 不同温度烧结的 Ce: YAG 荧光粉(a)和 Ce: YAG 苂光玻璃(b)的色坐标值

Fig. 2 CIE of Ce: YAG phosphors (a) and color conversion glasses (b) sintered at different temperatures 


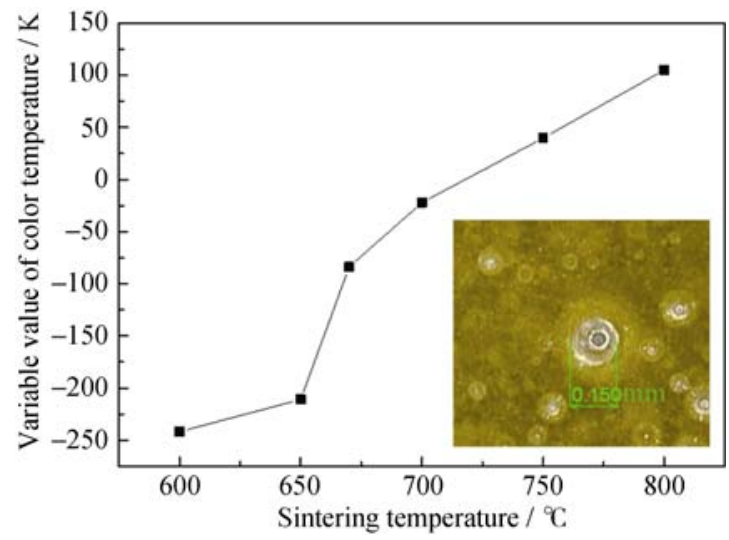

图 3 不同烧结温度下 $\mathrm{Ce}: \mathrm{YAG}$ 荧光玻璃与 $\mathrm{Ce}$ : $\mathrm{YAG}$ 荧光粉 的色温差值

Fig. 3 Variable value of color temperature between color conversion glasses and Ce: YAG phosphors sintered at different temperatures

\section{2 烧结性能研究}

为了分析烧结温度对荧光玻璃发光性能的影响 原因, 采用阿基米德法分别测试了 $600^{\circ} \mathrm{C}$ 和 $750^{\circ} \mathrm{C}$ 烧 结的荧光玻璃的密度, 其密度值分别为 2.970 和 $3.082 \mathrm{~g} / \mathrm{cm}^{3}$, 密度差值 $0.112 \mathrm{~g} / \mathrm{cm}^{3}$ 。密度测量结果 进一步证实低温烧结时荧光玻璃中存在气泡, 这会 影响苂光玻璃在 $460 \mathrm{~nm}$ 蓝光的激发光谱强度。

根据 Ce: YAG 的发光原理 ${ }^{[14]}$ 可知, 影响 $\mathrm{Ce}$ : YAG 的发光性能主要有两方面: $\mathrm{Ce}^{3+}$ 的含量和 $\mathrm{Ce}^{3+}$ 周围的晶体场环境。因此, 实验测试了不同温度烧 结的荧光玻璃的 XRD 和 XPS 图谱, 分析了苂光玻 璃中 $\mathrm{Ce}^{3+}$ 周围的晶体场环境和 $\mathrm{Ce}^{3+}$ 的含量的变化情 况。图 4 所示为不同温度保温烧结 $20 \mathrm{~min}$ 得到的 $\mathrm{Ce}: \mathrm{YAG}$ 苂光粉和 Ce: YAG 荧光玻璃的 XRD 图谱。 如图 4(a)所示, 对比 JCPDS 卡片 (00-033-0040), 不同温度烧结的荧光粉的 XRD 图谱基本没有变化, 说明 YAG 晶格结构没有变化。而不同温度烧结的苂
光玻璃的 XRD 图谱变化较大, 如图 4(b)所示, 在 $700^{\circ} \mathrm{C}$ 烧结时, $\mathrm{YAG}$ 衍射峰没有变化, $\mathrm{YAG}$ 晶格结构 没有被破坏，所以在 $560 \mathrm{~nm}$ 处获得了最强的发射光 谱, 而在 $750^{\circ} \mathrm{C}$ 烧结时, $\mathrm{YAG}$ 在 $2 \theta=33.3^{\circ}$ 处主峰强度 降低，导致苂光玻璃在 $560 \mathrm{~nm}$ 处发光强度减弱，直 到 $850^{\circ} \mathrm{C}$ 烧结时, 所有 $\mathrm{YAG}$ 衍射峰消失, YAG 晶格 结构被破坏, 苂光玻璃失去了发光性能。XRD 测试 结果与图 1 荧光光谱结果一致。

图 6 显示了 $700^{\circ} \mathrm{C}$ 和 $850^{\circ} \mathrm{C}$ 烧结的荧光玻璃的 XPS 图谱与拟合曲线图。图 5 为校准用的 $\mathrm{C} 1 \mathrm{~s}$ 谱图, 结合能为 $284.8 \mathrm{eV}$, 设备校准合格。通过查询标准 XPS 谱图数据库, $\mathrm{Ce}^{3+} 3 \mathrm{~d}_{5 / 2}$ 和 $\mathrm{Ce}^{3+} 3 \mathrm{~d}_{3 / 2}$ 的结合能分 别为 884 和 $902 \mathrm{eV}$, 而 $\mathrm{Ce}^{4+} 3 \mathrm{~d}_{5 / 2}$ 和 $\mathrm{Ce}^{4+} 3 \mathrm{~d}_{3 / 2}$ 的结合 能分别为 881.6 和 $899.9 \mathrm{eV}$ 。当 $\mathrm{Ce}$ 原子周围的化学 环境发生变化时，原子体系受到微扰，内层电子的 结合能大小可能会发生最大偏移量 $17.9 \mathrm{eV}^{[15]}$ 。对 $700^{\circ} \mathrm{C}$ 和 $850^{\circ} \mathrm{C}$ 烧结的两个荧光玻璃样品的曲线分 别进行分峰拟合, 如图 6(a)所示, $700^{\circ} \mathrm{C}$ 烧结样品的 4 个拟合峰分别为 $885.391 \mathrm{eV}\left(\mathrm{Ce}^{4+} 3 \mathrm{~d}_{5 / 2}\right), 890.518 \mathrm{eV}$ $\left(\mathrm{Ce}^{3+} 3 \mathrm{~d}_{5 / 2}\right), \quad 899.953 \mathrm{eV}\left(\mathrm{Ce}^{4+} 3 \mathrm{~d}_{3 / 2}\right), \quad 904.473 \mathrm{eV}$ $\left(\mathrm{Ce}^{3+} 3 \mathrm{~d}_{3 / 2}\right)$; 如图 6(b)所示, $850^{\circ} \mathrm{C}$ 烧结样品的 4 个拟 合峰分别为 $887.956 \mathrm{eV}\left(\mathrm{Ce}^{4+} 3 \mathrm{~d}_{5 / 2}\right), 891.148 \mathrm{eV}$ $\left(\mathrm{Ce}^{3+} 3 \mathrm{~d}_{5 / 2}\right), \quad 901.529 \mathrm{eV}\left(\mathrm{Ce}^{4+} 3 \mathrm{~d}_{3 / 2}\right), \quad 906.428 \mathrm{eV}$ $\left(\mathrm{Ce}^{3+} 3 \mathrm{~d}_{3 / 2}\right)$ 。还对拟合峰的面积进行计算, $\mathrm{Ce}^{3+}$ 的相 对含量用 $\mathrm{Ce}^{3+}$ 峰面积与 $\mathrm{Ce}^{3+}+\mathrm{Ce}^{4+}$ 峰面积比来估算, 如表 1 所示。表 1 数据表明, 当烧结温度从 $700^{\circ} \mathrm{C}$ 升 高到 $850^{\circ} \mathrm{C}$ 时, 苂光玻璃的 $\mathrm{Ce}^{3+}$ 含量从 $57.96 \%$ 降到 $17.67 \%, \mathrm{Ce}^{4+}$ 含量从 $42.04 \%$ 增加到 $82.33 \%$ 。 XPS 测试结果表明, 当烧结温度从 $700^{\circ} \mathrm{C}$ 升高到 $850^{\circ} \mathrm{C}$ 时, 一部分 $\mathrm{Ce}^{3+}$ 被玻璃基质氧化成 $\mathrm{Ce}^{4+}, \mathrm{Ce}^{3+}$ 含量减 少, 因而降低了荧光玻璃在 $560 \mathrm{~nm}$ 处的发光强度。 XPS 测试结果与图 1 苂光光谱结果一致。
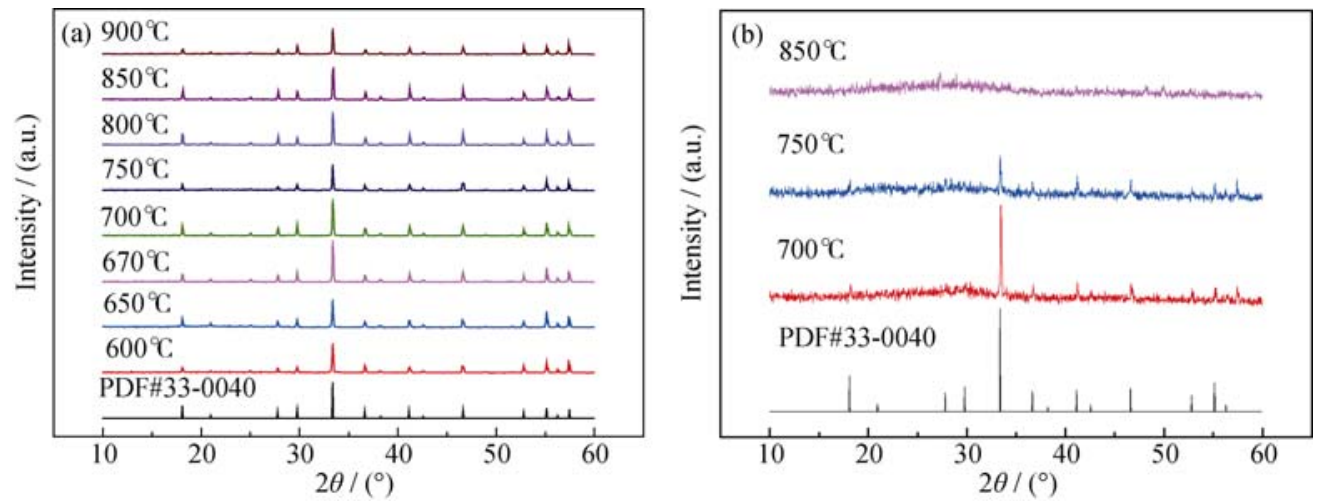

图 4 不同温度烧结的荧光粉(a)和荧光玻璃(b)的 XRD 图谱

Fig. 4 XRD patterns of Ce: YAG phosphors (a) and color conversion glasses (b) sintered at different temperatures 


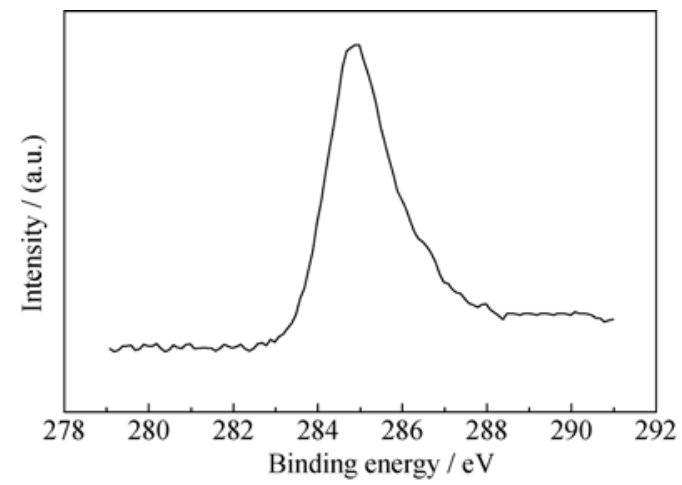

图 5 能谱仪设备校准用 $\mathrm{C} 1 \mathrm{~s}$ 的 XPS 谱图

Fig. 5 XPS spectrum of $\mathrm{C} 1 \mathrm{~s}$ for the equipment calibration

为了进一步分析玻璃基质对苂光粉的影响, 实 验测试了 $\mathrm{Ce}: \mathrm{YAG}$ 苂光粉, 硼硅玻璃基质和 $\mathrm{Ce}$ : YAG 苂光玻璃的 DSC 曲线, 如图 7 所示。从 DSC 曲线可以看出, 苂光粉在 $240^{\circ} \mathrm{C}$ 有个小吸热峰, 来 自于残余氢氧化物和碳酸盐沉淀吸热分解; 嗍硅

(a)

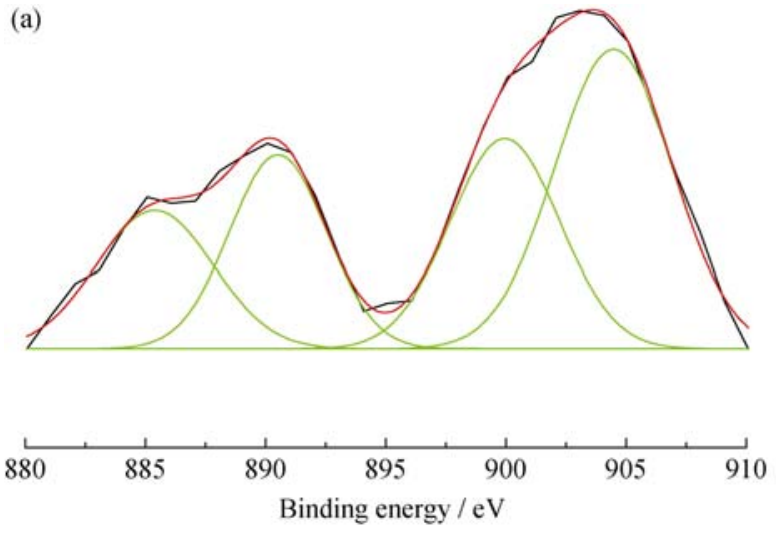

玻璃基质在 $500 \sim 700^{\circ} \mathrm{C}$ 有两个吸热峰，来自于玻璃 态 $\rightarrow$ 高弹态 $\rightarrow$ 粘流态转变, $700^{\circ} \mathrm{C}$ 有个结晶放热峰; 荧光玻璃在 $500^{\circ} \mathrm{C}$ 有个吸热峰, 在 $620^{\circ} \mathrm{C}$ 有个吸热峰, 与玻璃基质的吸热峰相似，也是来自于玻璃态 $\rightarrow$ 高 弹态 $\rightarrow$ 粘流态的转变; $810^{\circ} \mathrm{C}$ 有个较深放热峰, 根据 XPS 测试结果得出, 此放热峰可能是由于苂光粉在 玻璃基质中发生了氧化反应, $\mathrm{Ce}^{3+}$ 被氧化成 $\mathrm{Ce}^{4+}$ 造成 的, 文献[15-16]也研究了 $\mathrm{Ce}: \mathrm{YAG}$ 在高温氧化气氛 中 $\mathrm{Ce}^{3+}$ 易被氧化成 $\mathrm{Ce}^{4+}$ 。荧光玻璃中 $\mathrm{Ce}^{3+}$ 离子有效 发光数量减少, 因此超过 $850^{\circ} \mathrm{C}$ 烧结时, 苂光玻璃没 有激发谱峰 $\left(\mathrm{Ce}^{3+}: 4 \mathrm{f} \rightarrow 5 \mathrm{~d}\right)$ 和发射谱峰 $\left(\mathrm{Ce}^{3+}: 5 \mathrm{~d} \rightarrow 4 \mathrm{f}\right)$, 如图 1 所示。此外, 当温度超过 $700^{\circ} \mathrm{C}$ 时, 玻璃处在粘 流态，随着温度继续升高，玻璃液体的粘度也随之降 低, 导致玻璃液对苂光粉YAG 晶体的腐蚀加重, 破坏 了荧光粉的晶体结构, 如图 4(b)所示, 造成 $\mathrm{Ce}^{3+}$ 发光 环境改变, $\mathrm{Ce}^{3+}$ 在玻璃中的发光强度低于在 $\mathrm{YAG}$ 晶体 中的, 从而导致荧光玻璃的发光强度降低。

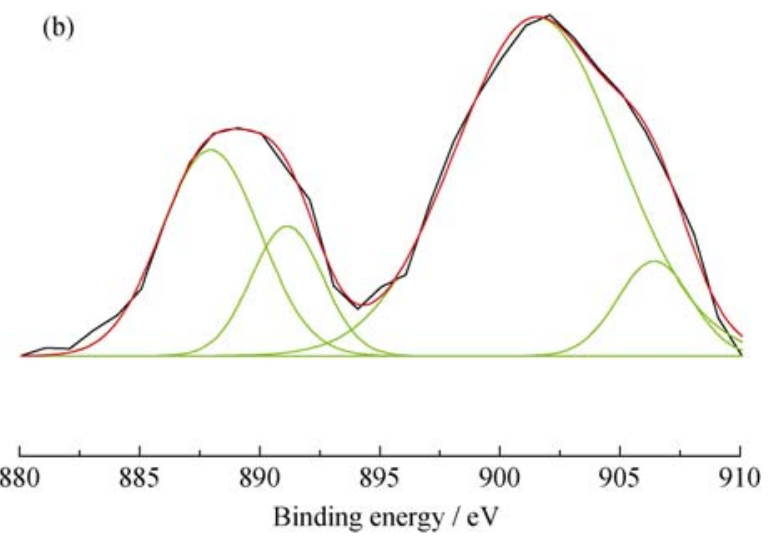

图 $6700^{\circ} \mathrm{C}(\mathrm{a})$ 和 $850^{\circ} \mathrm{C}(\mathrm{b})$ 烧结的荧光玻璃的 XPS 图谱与拟合曲线

Fig. 6 XPS spectra and fitting curves of Ce3d in color conversion glasses sintered at $700^{\circ} \mathrm{C}$ (a) and $850^{\circ} \mathrm{C}$ (b)

表 $1700^{\circ} \mathrm{C}$ 和 $850^{\circ} \mathrm{C}$ 烧结的荧光玻璃中 $\mathrm{Ce}^{3+}$ 相对含量

Table 1 Relative percentage of $\mathrm{Ce}^{3+}$ in the color conversion glasses sintered at $700{ }^{\circ} \mathrm{C}$ and $850^{\circ} \mathrm{C}$

\begin{tabular}{|c|c|c|c|c|c|c|}
\hline & Sintering temperature $/{ }^{\circ} \mathrm{C}$ & Peak/eV & FWHM/eV & Area /(a.u) & Sum/(a.u.) & Relative percentage $/ \%$ \\
\hline $\mathrm{Ce}^{4+} 3 \mathrm{~d}_{5 / 2}$ & \multirow{2}{*}{700} & 885.391 & 5.71 & 83433.797 & \multirow{2}{*}{204219.715} & \multirow{2}{*}{42.04} \\
\hline $\mathrm{Ce}^{4+} 3 \mathrm{~d}_{3 / 2}$ & & 899.953 & 5.36 & 120785.918 & & \\
\hline $\mathrm{Ce}^{3+} 3 \mathrm{~d}_{5 / 2}$ & \multirow{2}{*}{700} & 890.518 & 4.75 & 98702.643 & \multirow{2}{*}{281556.319} & \multirow{2}{*}{57.96} \\
\hline $\mathrm{Ce}^{3+} 3 \mathrm{~d}_{3 / 2}$ & & 904.473 & 5.75 & 182853.676 & & \\
\hline $\mathrm{Ce}^{4+} 3 \mathrm{~d}_{5 / 2}$ & \multirow{2}{*}{850} & 887.956 & 4.83 & 52640.837 & \multirow{2}{*}{195529.716} & \multirow{2}{*}{82.33} \\
\hline $\mathrm{Ce}^{4+} 3 \mathrm{~d}_{3 / 2}$ & & 901.529 & 8.00 & 142888.879 & & \\
\hline $\mathrm{Ce}^{3+} 3 \mathrm{~d}_{5 / 2}$ & \multirow{2}{*}{850} & 891.148 & 3.51 & 24062.383 & \multirow{2}{*}{41966.744} & \multirow{2}{*}{17.67} \\
\hline $\mathrm{Ce}^{3+} 3 \mathrm{~d}_{3 / 2}$ & & 906.428 & 3.62 & 17904.361 & & \\
\hline
\end{tabular}




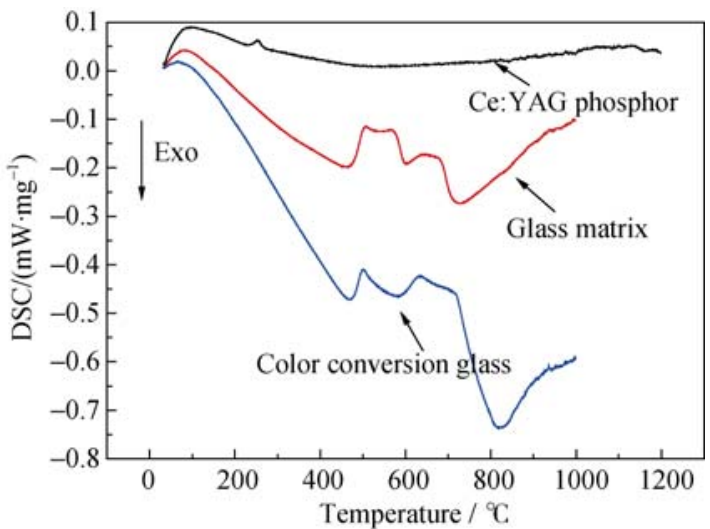

图 $7 \mathrm{Ce}: \mathrm{YAG}$ 荧光粉、嗍硅玻璃基质和 $\mathrm{Ce}: \mathrm{YAG}$ 苂光玻璃 的 DSC 曲线

Fig. 7 DSC curves of Ce: YAG phosphor, glass matrix and the color conversion glass

\section{3 结论}

实验研究了不同烧结温度制备的 Ce: YAG 嗍硅 基质荧光玻璃的发光性能, 发现烧结温度为 $700^{\circ} \mathrm{C}$ 时，玻璃基质刚进入粘流态，发光性能最佳，发射 光谱的峰值最强, 苂光玻璃的色坐标最接近荧光粉; 当烧结温度超过 $850^{\circ} \mathrm{C}$ 时, 玻璃基质破坏了荧光玻 璃的发光性能, 主要原因在于: 1) $\mathrm{Ce}^{3+}$ 被氧化成 $\mathrm{Ce}^{4+}$, 降低了苂光玻璃内 $\mathrm{Ce}^{3+}$ 的发光; 2)玻璃基质粘度 降低，加重了玻璃液对荧光粉 $\mathrm{YAG}$ 晶格结构的腐 蚀破坏, 改变了 $\mathrm{Ce}^{3+}$ 发光环境, 降低了苂光玻璃内 $\mathrm{Ce}^{3+}$ 的发光。结果表明，通过控制合适的烧结温度， 可以有效降低玻璃基质对荧光粉发光性能及色坐标 的劣化影响，提高苂光玻璃的发光性能和色坐标稳 定性能，从而推动荧光玻璃在 LED 封装中的应用。

\section{参考文献:}

[1] MAO Z Y, ZHU Y C, GAN L, et al. Novel white-light-emitting SiAlON-crystal/glass composite phosphor prepared by facile strategy for white light-emitting-diode. Materials Letters, 2012, 80(8): 63-65.

[2] NAKAMURA S. III-V nitride-based light-emitting diodes. Diamond and Related Materials, 1996, 5(3): 496-500.

[3] CRAWFORD M H. LEDs for solid-state lighting: performance challenges and recent advances. IEEE Journal of Selected Topics in Quantum Electronics, 2009, 15(4): 1028-1040.

[4] HUANG K C, HUANGY R, TING S Y, et al. Facile fabrication of spherical silicone encapsulant with Pt nanoparticles for applications as white light-emitting diode. Materials Letters, 2014, 134(7): 244-247.

[5] TSAI CC, WANG J, CHEN M H, et al. Investigation of Ce: YAG doping effect on thermal aging for high-power phosphor-converted white-light-emitting diodes. IEEE Transactions on Device and Materials Reliability, 2009, 9(3): 367-370.

[6] CHEN D Q, XIANG W D, LIANG X J, et al. Advances in transparent glass-ceramic phosphors for white light-emitting diodes - a review. Journal of the European Ceramic Society, 2014, 35(3): 859-869.

[7] ZHANG R, LIN H, YU Y L, et al. A new-generation color converter for high-power white LED: transparent $\mathrm{Ce}^{3+}$ : YAG phosphor-in-glass. Laser \& Photonics R, 2014, 8(1): 158-164.

[8] BORRELLI N F, LAMBERSON L A, MORENAR M, et al. Phosphor containing glass frit materials for LED lighting applications. US, C03C3/066, US8822032. 2014.09.02.

[9] CHEN H, LIN H, XU J, et al. Chromaticity-tunable phosphor-in-glass for long-lifetime high-power warm w-LEDs. Journal of Materials Chemistry C, 2015, 3(31): 8080-8089.

[10] SEGAWA H, HIROSAKI N, OHKI S, et al. Exploration of zinc phosphate glasses dispersed with Eu-doped SiAlON for white LED applications. Optical Materials, 2013, 35(12): 2677-2684.

[11] ZHANG Y, LIU S, XU H J, et al. Preparation and performance of Ce: YAG phosphor-in-glass. Journal of Inorganic Materials, 2015, 30(6): 588-592.

[12] REN L J, LEI X H, DU X Q, et al. Effect of $\mathrm{Eu}_{2} \mathrm{O}_{3}$ concentration on luminescent properties of $\mathrm{Ce} / \mathrm{Tb} / \mathrm{Eu}$ co-doped calcium borosilicate glass for white LED. Journal of Luminescence, 2013, 142(8): $150-154$.

[13] 2015/863/EU(ROHS 2.0), the Restriction of the use of certain hazardous substances in electrical and electronic equipment.

[14] 张中太, 张俊英. 无机光致发光材料及应用, 2 版. 北京: 化学 工业出版社, 2011: 93.

[15] WANG L M, ZHUANG L Q, HUANG Y X, et al. Application of XPS in semi-quantitative estimation of $\mathrm{Ce}^{3+}$ in YAG: $\mathrm{Ce}^{3+}$ phosphor. Journal of East China University of Science and Technology (Natural Science Edition), 2015, 41(4): 484- 488.

[16] 陈珍霞. LED 用低熔点发光玻璃的研究. 武汉: 武汉理工大学 硕士学位论文, 2010 . 